\title{
Time-resolved Raman spectroscopy for in situ planetary mineralogy
}

\author{
Jordana Blacksberg, ${ }^{1, *}$ George R. Rossman, ${ }^{2}$ and Anthony Gleckler ${ }^{3}$ \\ 'Jet Propulsion Laboratory, California Institute of Technology, Pasadena, California 91109, USA \\ ${ }^{2}$ California Institute of Technology, Division of Geological and Planetary Sciences, Pasadena, California 91125, USA \\ ${ }^{3}$ GEOST, Inc., Tucson, Arizona 85741, USA \\ *Corresponding author: jordana.blacksberg@jpl.nasa.gov
}

Received 12 April 2010; revised 20 July 2010; accepted 12 August 2010;

posted 13 August 2010 (Doc. ID 126635); published 8 September 2010

\begin{abstract}
Planetary mineralogy can be revealed through a variety of remote sensing and in situ investigations that precede any plans for eventual sample return. We briefly review those techniques and focus on the capabilities for on-surface in situ examination of Mars, Venus, the Moon, asteroids, and other bodies. Over the past decade, Raman spectroscopy has continued to develop as a prime candidate for the next generation of in situ planetary instruments, as it provides definitive structural and compositional information of minerals in their natural geological context. Traditional continuous-wave Raman spectroscopy using a green laser suffers from fluorescence interference, which can be large (sometimes saturating the detector), particularly in altered minerals, which are of the greatest geophysical interest. Taking advantage of the fact that fluorescence occurs at a later time than the instantaneous Raman signal, we have developed a time-resolved Raman spectrometer that uses a streak camera and pulsed miniature microchip laser to provide picosecond time resolution. Our ability to observe the complete time evolution of Raman and fluorescence spectra in minerals makes this technique ideal for exploration of diverse planetary environments, some of which are expected to contain strong, if not overwhelming, fluorescence signatures. We discuss performance capability and present time-resolved pulsed Raman spectra collected from several highly fluorescent and Mars-relevant minerals. In particular, we have found that conventional Raman spectra from fine grained clays, sulfates, and phosphates exhibited large fluorescent signatures, but high quality spectra could be obtained using our time-resolved approach. (C) 2010 Optical Society of America

OCIS codes: $\quad 300.6500,300.6450,300.6280,300.6190$.
\end{abstract}

\section{Introduction}

Since its discovery more than 80 years ago, and elevation 30 years later with the invention of the laser, Raman spectroscopy has evolved into the technique of choice for the in situ exploration of planetary bodies because it addresses a primary goal of mineralogical analysis: determination of structure and composition. With their high spectral and spatial resolution, laser Raman spectrometers for both surface and subsurface analysis are currently under develop-

0003-6935/10/264951-12\$15.00/0

(C) 2010 Optical Society of America ment for a diverse set of planetary targets, including Mars and its moons Phobos and Deimos, Venus, Earth's moon, and asteroids. Localized analyses of planetary surfaces using laser Raman spectroscopy complement global satellite remote sensing using visible-infrared imaging from orbit and are the prime consideration for preselection of rock samples prior to caching for potential sample return missions.

\section{A. Comparison of Techniques}

Several techniques can be used for mineralogical analysis of planetary bodies. From orbit, spectacular results have been achieved using visible-IR imaging spectroscopy of Mars and the Moon. Two prime 
examples are the Compact Reconnaissance Imaging Spectrometer for Mars (CRISM) on the Mars Reconnaissance Observer (MRO) and the Moon Mineralogy Mapper (M3) on the Lunar Reconnaissance Orbiter (LRO). The resulting global mineralogy maps can be placed in detailed context when combined with high resolution imaging provided by instruments such as HiRISE on MRO. Additionally, gamma ray mapping of hydrogen (e.g., on Mars Odyssey) has led to the conclusion that huge amounts of water exist over a large percentage of Mars.

Guided by orbital data, a host of in situ techniques summarized in Table 1 can provide mineralogical information locally within a well-defined geological context. Of these techniques, only x-ray diffraction (XRD) and Raman spectroscopy can definitively identify nearly all crystalline minerals based upon unique narrowband spectroscopic features. For XRD, sample collection is achieved through the use of a robotic arm that delivers a rock fragment that is typically powdered for analysis. Laser Raman spectroscopy offers the important advantage that it is nondestructive to the sample, can be operated meters away from the contaminated area of the spacecraft (e.g., on a rover arm), and is directed to a specific target sample within the context of its natural mineral setting. It is the directional, intense, coherent nature of the laser that provides the important ability to focus down to a very small spot size comparable to mineralogical grains. This provides the foundation for two-dimensional Raman mapping.

Although a highly successful method for mineralogical analysis from orbit, visible to near-IR spectroscopy is inherently low-resolution because it relies on the solar reflectance spectra of solid-phase materials. Hence, much of the subtlety of this technique is found in painstaking analysis of low-resolution, poor contrast features. Laser Raman spectroscopy [1], however, is inherently a high spectral resolution technique, because the returned Raman spectra show extremely narrow vibrational features. For a gas, they can be considered infinitely narrow $\left(<0.03 \mathrm{~cm}^{-1}\right)$, but even for a solid, the Raman spectral features are considered extremely sharp $\left(\sim 3-10 \mathrm{~cm}^{-1}\right)$. The use of a moderately high resolution spectrometer can therefore provide unique Raman fingerprints for unambiguous mineral identification. Laser Raman spectroscopy from a mineral sample under laser excitation relies on the measurement of scattered radiation, which differs in wavelength from the incident beam as a result of interaction with phonons. Because each band in a Raman spectrum represents interaction of the incident light with a vibrational mode in the crystal, it is highly specific for a given mineral and can be used for identification and structural characterization of unknown samples. An added benefit is that the observed Raman shifts (defined as the shifts of Raman peaks in wavenumber from the laser line) are nearly independent of the excitation wavelength, greatly simplifying the development of Raman data- bases, which can draw from data taken using any excitation wavelength.

\section{B. Mineralogy of Mars and Venus}

As a result of the impressive orbital and in situ data added over the past decade or so, we have come to understand Mars as a diverse body thought to have had two distinct geological histories. The early history may be characterized by a water-rich environment enabling the formation of minerals, such as clays. This neutral-to-alkaline environment is thought to have evolved to a sulfurous acidic environment as aqueous activity diminished and volcanic activity increased. Most of the aqueous alteration is likely to have occurred in the early Martian history with the formation of minerals such as goethite; jarosite; $\mathrm{Fe}-, \mathrm{Mg}-$, and Ca- sulfates; hydrated sulfates (e.g., kieserite); phyllosilicates (e.g., montmorillonite clays); and Fe-, $\mathrm{Mg}-$, and Ca- carbonates [2]. It is this depiction of earlier Martian conditions that also leads us to the exciting prospect of discovering evidence of past life on Mars.

A number of richly successful orbital missions have laid the foundation for in situ exploration on Mars [3]. Our current understanding of Martian planetary mineralogy is in large part inferred through the analysis of data from the Thermal Emission Spectrometer (TES) on the Mars Global Surveyor (launched in 1996), the Thermal Emission Imaging System (THEMIS) and Gamma Ray Spectrometer (GRS) on the Mars Odyssey (launched in 2001), Visible and Infrared Mineralogical Mapping Spectrometer (OMEGA) on the Mars Express (launched 2003), and, most recently, the Compact Reconnaissance Imaging Spectrometer for Mars (CRISM) [4] on the Mars Reconnaissance Orbiter (MRO) (launched 2005) and its complementary HiRISE camera [5]. It is through these missions that we have evidence for the overarching basaltic nature of Mars, and the discovery of altered regions containing minerals such as sulfates, phyllosilicates, iron oxides, zeolites, and water ice.

Although Mars is primarily basaltic with typically only a weak degree of alteration, much of our interest lies in the altered regions. It is these regions that we aim for with landers carrying in situ instruments. Evidence for past liquid water on Mars comes, in part, from evidence of sulfate-rich sediments that contain minerals such as jarosite, calcium and magnesium sulfates [6], and hematite spheres, indentified spectroscopically with Mini-TES on the Mars Exploration Rover (MER) [7], that led to the realization that some of the sandstones were derived from an evaporitic source [8]. The presence of goethite at the Columbia Hills site is of great importance because, unlike hematite, this mineral can only form in the presence of water. However, with the exception of Fe-bearing sulfates, hematite, and goethite identified by the MER Mössbauer instrument, there have been no alteration phases unambiguously identified by in situ instruments to date [2]. 


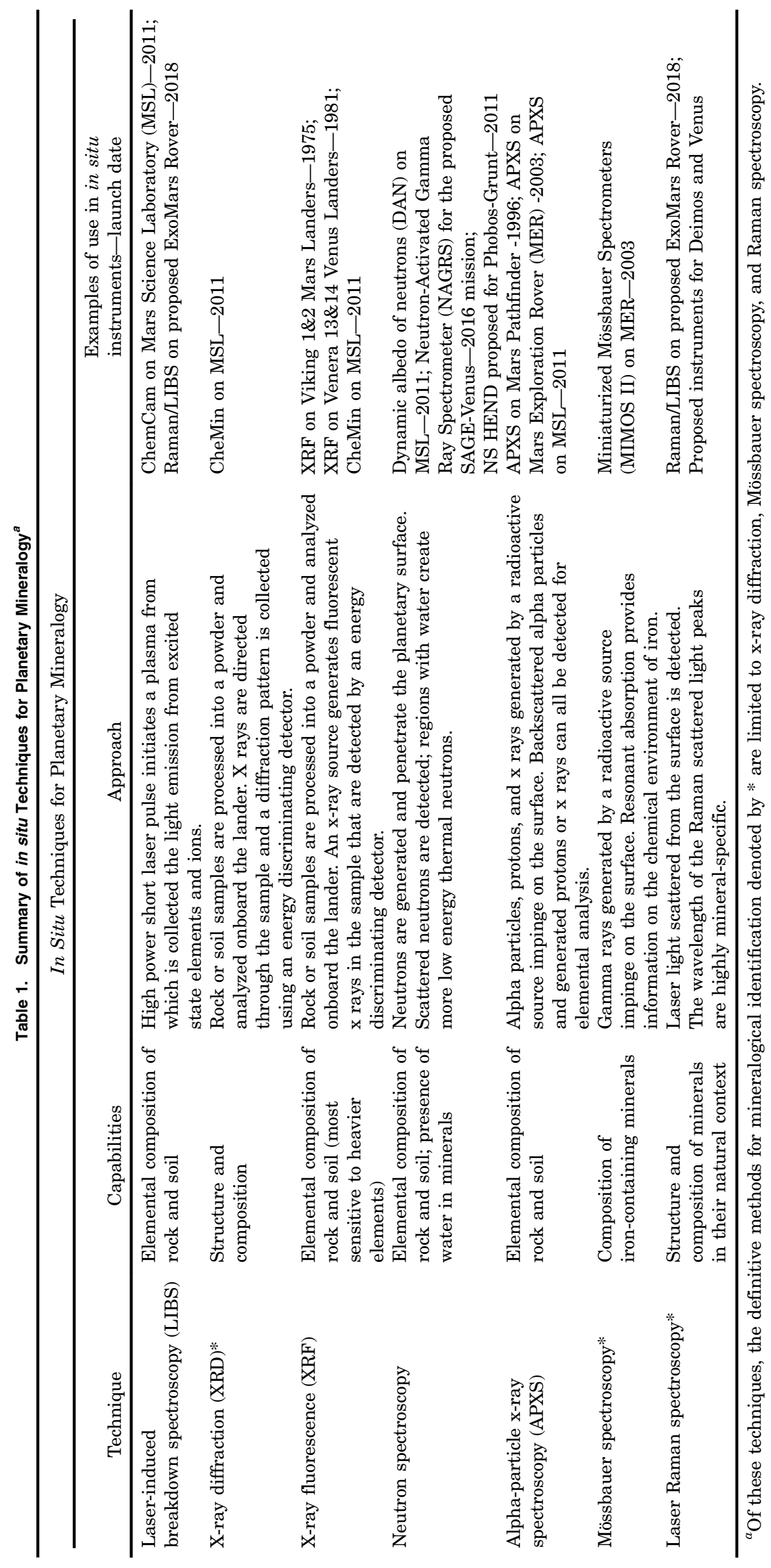


The next planned in situ mission to Mars, the Mars Science Laboratory [(MSL) to be launched in 2011] is expected to change this fact [9]. It will carry the ChemCam and CheMin instruments for both elemental and mineralogical analysis, respectively, with the Sample Analysis at Mars (SAM) suite providing detailed isotopic analysis in pyrolysis and combustion experiments. Because of the desire to study an altered region with the MSL, the selection of the landing site is crucial. The orbital data has therefore been essential in identifying the four potential landing sites at Mawrth Vallis, Gale Crater, Eberswalde Crater, and Holden Crater. The MSL will use the high resolution MastCam and the laser induced breakdown spectroscopy (LIBS) or ChemCam to rapidly survey the geology in the vicinity of the rover, and the neutron spectrometer to survey for water-containing minerals. The samples of greatest interest will be processed into a powder and delivered to CheMin (XRD/XRF) and SAM. The data from CheMin will be the first truly definitive mineralogical data outside of that obtained on iron-containing minerals using Mössbauer.

In situ Mars exploration beyond MSL is expected to undertake the challenging goal of sample return. Because the quantity of rock and soil that could be returned is limited (likely od the order of several hundreds of grams, total), this would require precise in situ instruments that could definitively identify interesting samples for caching and possible return. Raman spectroscopy is the technique of choice because, unlike XRD, it does not require sample preparation, and Raman spectra can be acquired on multiphase rock and soil samples as they are found in nature, preserving rock fabrics and textures. The raw spectra provide unique fingerprints that can often be easily interpreted without recourse to complex spectral deconvolution. The power of Raman spectroscopy to unambiguously identify minerals, for example in a mixed phase Mars meteorite, has been well-demonstrated [10]. Raman spectroscopic mapping in conjunction with complementary techniques such as imaging, elemental analysis (e.g., LIBS), and ultraviolet fluorescence (for organic detection) [11] would provide a strong comprehensive set of measurements for successful identification of minerals and trace components.

Relative to Mars, very little is known about the surface of Venus. The planet is surrounded by a dense carbon dioxide atmosphere with a pressure 90 times that of Earth. In addition, the surface temperature is around $450{ }^{\circ} \mathrm{C}$, making it very difficult for a lander or probe to survive more than a few hours. The prospect of a rover spending months or years on the surface, as has been the model for Mars, is highly unlikely. If we are to plan for mineralogical analysis on the Venus surface, rapid acquisition of spectra would be crucial. The XRD experiment (CheMin) on the Mars Science Laboratory will typically require hours for sample preparation and integration times of about10 $\times h$ for a single measurement. It is there- fore prohibitively long for Venus without further modification. On the other hand, Raman spectroscopy is a prime candidate for rapid mapping. By eliminating the need for extensive sample preparation, we could hope to survey many rock and soil samples during the short course of a landed mission, either for in situ analysis or potential sample return selection.

Our current knowledge of the surface of Venus [12] is derived from a series of Russian Venera and Vega probes from 1970 to 1985, which returned images and high resolution radar maps, as well as x-ray fluorescence (XRF) spectra of rock and soil samples. The Venera-Vega landing sites were comprised of soil and finely bedded rock, with evidence suggesting a primarily mafic surface composed of tholeiitic basalts and alkaline basalts. The Magellan mission in 1990 obtained radar images of the Venera-Vega landing sites, piecing together after the fact that these missions explored what are described as plains with wrinkle ridges. Magellan has also led to the identification of the tessera terrain as the first candidate for future in situ analysis and/or sample return. Tessera, on which no spacecraft has landed to date, is morphologically diverse with ridges and grooves, and it is expected to contain the oldest material recognized on Venus. Our knowledge of Venus mineralogy is based primarily on modeling of the XRF data, indicating high concentrations of silicon, aluminum, iron, magnesium, and calcium oxide. Unfortunately, XRF does not provide information on lighter elements, and therefore, hydrogen content, and thus hydration in minerals, is unknown. Modeling of minerals under Venus conditions suggests the stability of hydrated minerals such as hydrous silicates, as well as $\mathrm{OH}^{-}$, containing nominally anhydrous minerals such as pyroxenes [13]. Because this subject of mineralogical alteration and weathering in the Venus environment is of great interest, it perhaps suggests the need to get below the surface to access unweathered materials for analysis.

\section{Mineralogy of the Terrestrial Moons and Asteroids}

The Earth's moon and the moons of Mars (Phobos and Deimos) are the only satellites of terrestrial planets in our solar system. The origin, composition, and evolution of the Martian moons are still very much in question with various theories, including the capture of asteroids into the Martian orbit [14]. In addition, many asteroids are thought to be surviving protoplanets and planetesimals, and can act as windows into various stages of the formation of the solar system. A planned sample return mission to the Mars moon Phobos (Phobos-Grunt) in 2011 by the Russian Space Agency [15] aims to clarify some of the questions of its nature and origin, providing a more expansive understanding of terrestrial moons, asteroids, and the formation of the planets. In addition to returning samples to Earth, that mission includes an in situ lander with a robotic arm that will scoop samples, with elemental/mineralogical analysis relying on 
techniques such as alpha particle x-ray spectroscopy (APXS), neutron spectrometry, and mass spectrometric analysis of pyrolyzed samples. For the second Mars moon, Deimos, NASA is considering proposals to land on the surface with laser Raman spectroscopy playing a key role in mineralogical assessment.

Our understanding of the geology of the Earth's moon comes from a long history of Earth-based telescopic observations, orbiter observations, and direct sampling. Lunar sample return from the Earth's moon has been successfully accomplished by a series of manned (Apollo, 1969-1972) and unmanned autonomous (Soviet Luna, 1970-1976) missions. Two distinct regions have been identified: the highlands, which are anorthositic in composition, and the dark maria, which are basaltic in nature. The geology of numerous other regions is not yet well-understood. An example is the South Pole Aitken basin, the oldest, largest, and deepest impact feature on the Moon. It contains mineralogy that is expected to vary greatly from what has been found at the Apollo landing sites. This was confirmed by spectroscopic data acquired by Galileo, Clementine, and Lunar Prospector missions. This region is therefore of great interest for future in situ and potential sample return missions.

Raman spectroscopy has already been proven as a successful technique for determining the mineralogy, structure, and composition of lunar rocks, soils, and glasses returned to Earth by the Apollo missions [16]. The primary constituents in lunar samples, such as feldspar, pyroxene, and olivine, have been easily identified using Raman spectroscopy. Interestingly, fluorescence interference was observed in some of the lunar samples, pointing to the potential value of a time-resolved approach. Potential future in situ missions to the Earth's moon and the moons of Mars could take advantage of Raman spectroscopy, particularly for studying permanently shadowed areas, such as those found in the lunar South Pole. In such regions, passive visible-IR reflectance spectrometry could not be used, and an active source (laser) would likely be necessary to obtain useful spectroscopic data from either enhanced visible-IR or from laser Raman interrogation.

Small and primitive planetary bodies like asteroids are thought to be representative of the early stages of solar system formation. In situ mineralogical analysis of these bodies, while of great interest, would present additional challenges associated with landing in a very low gravity environment of unknown topography. This underscores the need for preliminary gravity and topographic mapping to help lower the risk of approaching and touching down onto these low-g bodies. Furthermore, if laser Raman instrumentation (e.g., focusing optical head) must be brought to surface samples using a robotic arm, a sampling strategy must be developed for low-g implementation, which could mean challenging ground-testing and verification before launch.

\section{Next Generation Laser Raman Spectrometers}

There are two types of Laboratory Raman spectrometers in widespread use: dispersive Raman and Fourier Transfer (FT)-Raman. Dispersive Raman spectrometers typically operate with visible excitation sources, fixed gratings, no moving parts, and large-format detectors such as CCDs. FT-Raman spectrometers typically operate with infrared laser sources and movable mirrors, which allow for a variable path length and the generation of an interferogram collected on a single element detector such as Ge or InGaAs. Of the dispersive Raman spectrometers, multichannel dispersive systems have surpassed single channel systems due to technological advancements, particularly in low-noise large-format CCD detectors. Multichannel spectrometers offer faster collection speeds and higher sensitivity, and are often shot-noise limited, providing the highest achievable signal-to-noise ratio (SNR). CW dispersive green Raman spectroscopy is commonly used in the laboratory for identification and characterization of minerals. For example, the RRUFF project [17] was established to create a complete set of high quality spectral data from well-characterized mineral samples to be used as standards for Raman fingerprinting. Much of the data was acquired using CW green Raman spectroscopy . However, for many minerals, Raman spectra are difficult or impossible to acquire using a CW green source due to a large interfering fluorescence signature. The fluorescence problem was addressed in 1986 by the introduction of the FTRaman spectrometer with an infrared laser excitation source (commonly $1064 \mathrm{~nm}$ ) [18,19]. A primary advantage of FT-Raman is that it greatly reduces the number of cases in which fluorescence interference is problematic, since infrared typically does not provide enough energy to excite fluorescence. It has gained popularity as a laboratory technique and has proven particularly useful for high resolution characterization of organic and biological materials, which often contain large fluorescence signatures when excited in the visible $[20,21]$. This advantage is often enough to justify using $1064 \mathrm{~nm}$ excitation, despite the significantly reduced signal level that comes with using long wavelength excitation (according to the $1 / \lambda^{4}$ dependence of the Raman return). Another recent development designed to combat fluorescence has been the near-IR dispersive spectrometer [22]. When combined with low noise detectors, dispersive spectrometers offer inherently larger SNRs than FT-spectrometers. However, the sensitivity of CCD detectors is too low at $1064 \mathrm{~nm}$ to be competitive with FT-Raman. An approach using dispersive spectrometers and shorter wavelength near-IR excitation (700-800 nm) works to reduce fluorescence in many cases, although not as significantly as $1064 \mathrm{~nm}$. Because each method offers its inherent advantages, the choice of spectrometer design will be sample dependent. For minerals, the use of IR excitation has yielded mixed results. In many cases, fluorescence interference from rare earth elements 
has been observed using a $1064 \mathrm{~nm}$ excitation leading to ambiguous results [23-25]. In addition, the fluorescence return is expected to be enhanced at low temperature, an important consideration for Mars and other cold bodies.

For several reasons, we have chosen a timeresolved approach for planetary mineralogy, rather than an infrared Raman approach to overcome fluorescence. First, the use of a green pulsed laser allows us to broaden the scope of our instrument to obtain not only Raman, but also complementary fluorescence, and potentially LIBS information. Second, the higher Raman return for green compared to infrared allows for faster collection times and lower laser power. And last, although Fourier transform IR (FTIR) systems have been designed for planetary missions (e.g., the mini-TES on the MER), dispersive systems offer the advantage of containing no moving parts.

Fluorescence interference is likely to be problematic on Mars, as evidenced by Raman measurements of Mars meteorites. For example, nearly half of the 362 Raman spectra taken on the Zagami Mars meteorite at $532 \mathrm{~nm}$ yielded no Raman information due to fluorescence [26]. Many minerals found on Mars are known to fluoresce strongly on Earth-for example, clays and sulfates. Because so little is still known about Venus mineralogy, it is difficult to assess the extent to which fluorescence interference would be a concern for Raman spectroscopy on Venus. As one example, modeling suggests the possible presence of hydrous minerals, such as tremolite on Venus [27], and these minerals have been known to fluoresce on Earth. Because of the risks associated with unknown mineralogy, it would be prudent to plan for an environment that includes fluorescence.

Raman scattering occurs instantaneously in time, while fluorescence occurs on longer time scales. Minerals can contain as many as 25 luminescence centers, each with different decay times from nsec to msec $[28,29]$. We use the distinct time scales of Raman and fluorescence to separate the spectra. In doing so, we can collect fluorescence-free Raman spectra using traditional visible laser excitation $(532 \mathrm{~nm})$ and simultaneously measure time-resolved fluorescence spectra for the detection of trace ionic components. Through the combined use of these techniques, the probability of successful mineral identification is greatly increased.

Time-resolved pulsed Raman spectroscopy is ideal for fluorescence rejection, since the Raman signal is collected simultaneously with the laser pulse, and photons emitted after the laser pulse can be rejected. Laser pulse widths of the order of $10 \mathrm{~ns}$ are typically combined with an intensified charge-coupled device (ICCD) based gated detection system [29]. By gating so as to detect only photons emitted during the laser pulse, the majority of the fluorescence signal is rejected. Rather than just reject the longer duration fluorescence with a time-gated cutoff, it would be preferable to collect and record the full time history of the Raman and fluorescence signals, and this can be captured with high temporal resolution ( 1 psecs) streak cameras [30-32]. Spectra collected with a streak camera are swept across a CCD using high speed voltage ramps in a streak tube. The result is a three-dimensional plot of intensity versus frequency and time. By using the entire time evolution rather than a fixed gate, it becomes possible to view Raman and fluorescence simultaneously. Other effects, such as plasma and LIBS related effects, can be seen, as well, if the energy density at the sample is high. The streak camera is therefore an invaluable tool for identifying and characterizing complex mineral samples as they are found in nature, containing both Raman and fluorescence centers.

\section{E. Challenges of Developing On-Surface Planetary Spectrometers}

As successful Apollo lunar missions illustrated, the elemental and mineralogical analysis of samples returned from planetary bodies can be made with extremely high accuracy and precision using state-ofthe-art spectrometers of various types (spectrometers based on mass, Raman, neutron, x-ray, alpha-particle, Mossbauer, etc.) housed in laboratories throughout the world. These instruments are, by definition, very large, consume high power, and often take up one or several rooms in size. Also, despite significant instrument automation, they usually require several staff members and students to operate and have associated large computing facilities for data processing and analysis.

In situ planetary instruments face challenges that greatly exceed even the challenges of developing in-space Earth orbiting instruments. First, the basic instrument design must be applicable to a diverse set of target bodies, some relatively nearby, such as Mars or Venus, and some a long way out, such as Saturn's moon Titan, or distant asteroids. Individual bodies may be associated with extreme pressures or temperatures-absolute and diurnal or seasonal changes. Because of restricted payload masses for these ambitious missions, planetary in situ instruments must be very small (a few $\mathrm{kg}$, at most), consume low power ( $20 \mathrm{~W}$ or less), take up low volume (a few liters, at most), and be highly automated from instrument turn-on to data transmission. Flight instrument practices by both NASA and other international agencies require environmental and operational testing that exceeds the expected planetary environment. The instrument must be designed to be readily testable (environmental and vibration), easily calibrated, and, in most cases, be able to withstand pyrotechnic shock, launch loads, planetary protection requirements, and radiation levels that strongly depend on target body and mission duration.

To develop a small, robust instrument that can survive all of the above described demands while achieving measurement capability comparable to state-of-the-art laboratory instruments will not be possible. Furthermore, long integration times to enhance signal-to-noise ratios may not be possible 
(e.g., touch-and-go analysis of asteroids or Venus surface measurements). However, there is an important role for laser Raman spectrometers, not only in onsurface mineralogical analysis, but also rock preselection prior to sample return, and various efforts have produced instruments that are feasible for planetary missions. Key subsystems of potential risk are the laser source itself and signal detector, and their flight qualification, with the receiving optics, optical train, and electronics considered more technically mature for flight. Estimates by JPL engineers for a time-gated green laser Raman spectrometer for a future Mars mission, for example, suggest that a complete instrument with a $5 \mu \mathrm{J} /$ pulse laser and solid-state detector could weigh only $3 \mathrm{~kg}$ total mass, consume $15 \mathrm{~W}$ of power, and take up only $1 L$ in volume. This compares well with existing in situ instruments for Mars, including those described in Table 1.

\section{Experiment}

\section{A. Experimental Setup}

A schematic of our time-resolved Raman instrument is shown in Fig. 1. A $532 \mathrm{~nm}$ passively $Q$-switched pulsed microchip laser (Arctic Photonics) delivers a $5 \mu \mathrm{J}$ pulse over $\sim 800 \mathrm{ps}$ at a repetition rate of

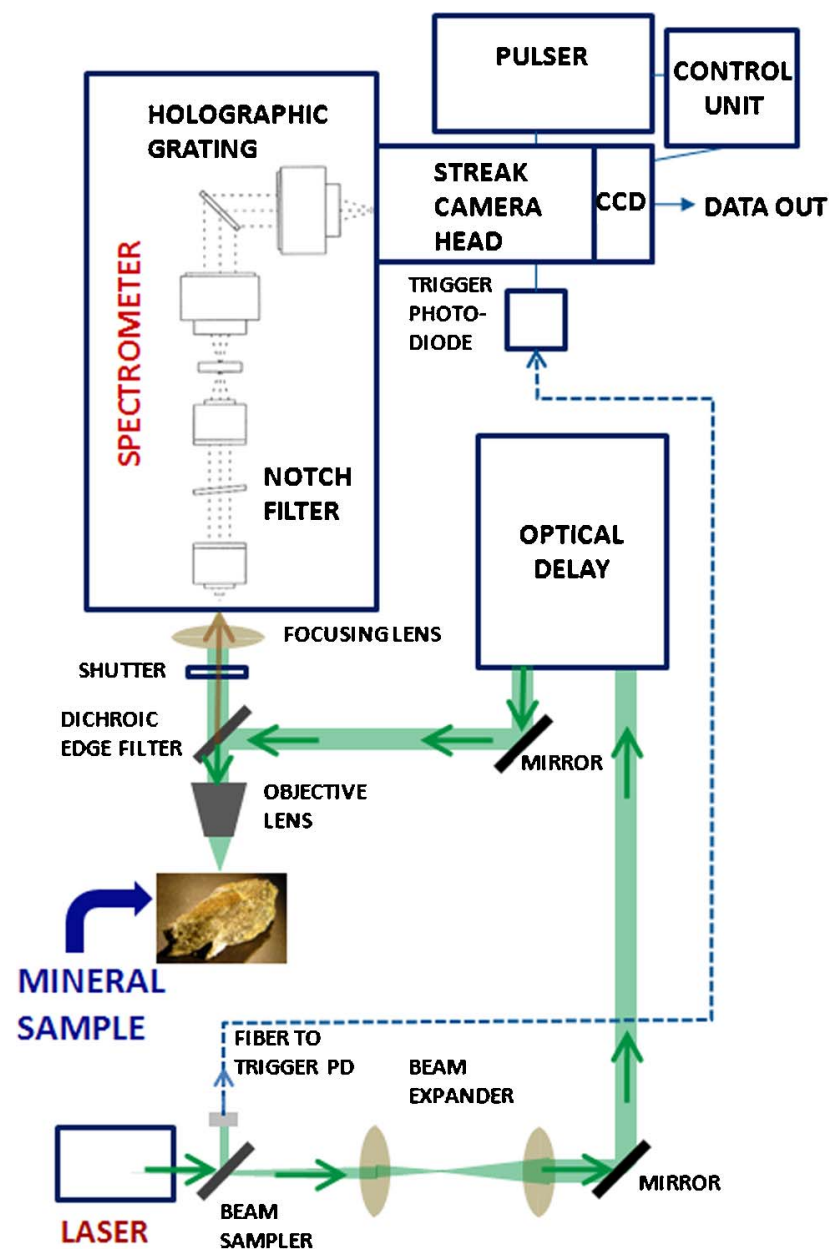

Fig. 1. (Color online) Schematic diagram of the time-resolved Raman instrument.
$1 \mathrm{kHz}$. A small portion $(<1 \%)$ of the beam is sampled and delivered to a trigger photodiode, which triggers the streak camera to sweep. The main beam goes through an optical delay line timed to ensure that the Raman return is collected and synchronized with the camera. A dichroic edge filter reflects the laser light to an objective lens, which focuses the beam onto the mineral sample. The Raman/fluorescence return passes back through the dichroic and is focused onto the input slit of a modified Kaiser Optical Holospec spectrometer. Inside the spectrometer, a holographic notch filter cuts out the laser light again by $\sim 6$ orders of magnitude. The light is focused onto a vertical slit prior to passing through a holographic grating, which spectrally disperses the light horizontally onto a $10 \mathrm{~mm}$ wide, $75 \mu \mathrm{m}$ high slit that defines the entrance to a custom Axis Photonique streak camera using a Photonis P925 streak tube.

The streak camera collects data in synchroscan mode in which the laser is synchronized to the sweep electronics. The pulser electronics sweeps the spectrum vertically over a chosen time base, which can be varied from $4 \mathrm{~ns}$ to $500 \mu \mathrm{s}$. The streak camera repeatedly streaks small pulses across the phosphor screen, and the CCD integrates these pulses. The output on the CCD is a three-dimensional image with the spectrum on one axis, time on another axis, and intensity on the third axis. The benefit of this approach is that a small and inexpensive low pulse energy laser (microchip laser) can be used, and that we do not incur CCD read noise with every pulse, which significantly improves SNR. The front-end gain of the streak camera coupled with the low dark current and read noise of the CCD results in an extremely low total measured noise of $<2$ photons per minute. Because only the return is synchronized with the laser, background light is eliminated (allowing for daylight operation) along with unwanted fluorescence. As a result, a pulsed system operating at the same average laser power as a CW system can achieve a given Raman peak SNR in a shorter collection time.

In our setup, an objective lens is used to focus the beam onto the mineral sample. The Raman return is collected in the $180^{\circ}$ configuration back through the objective lens. A set of objective lenses of $4 \times, 10 \times$, and $40 \times$ were used. The data presented here were acquired with the $40 \times$ objective, which has the highest NA, but also the shortest working distance.

Frequency calibration was performed using cyclohexane and acetonitrile standards. Radiometric analysis using cylcohexane as a standard [33] confirmed that the Raman return detected at the CCD is $>60 \%$ of the expected Raman return from the sample within the acceptance angle of the spectrometer. This is reasonable, considering the losses in the optical chain after the sample.

\section{Results and Discussion}

A typical streak camera image of calcite is shown in Fig. 2(a). The Raman peaks are simultaneous with the laser pulse and therefore appear as $800 \mathrm{ps}$ 

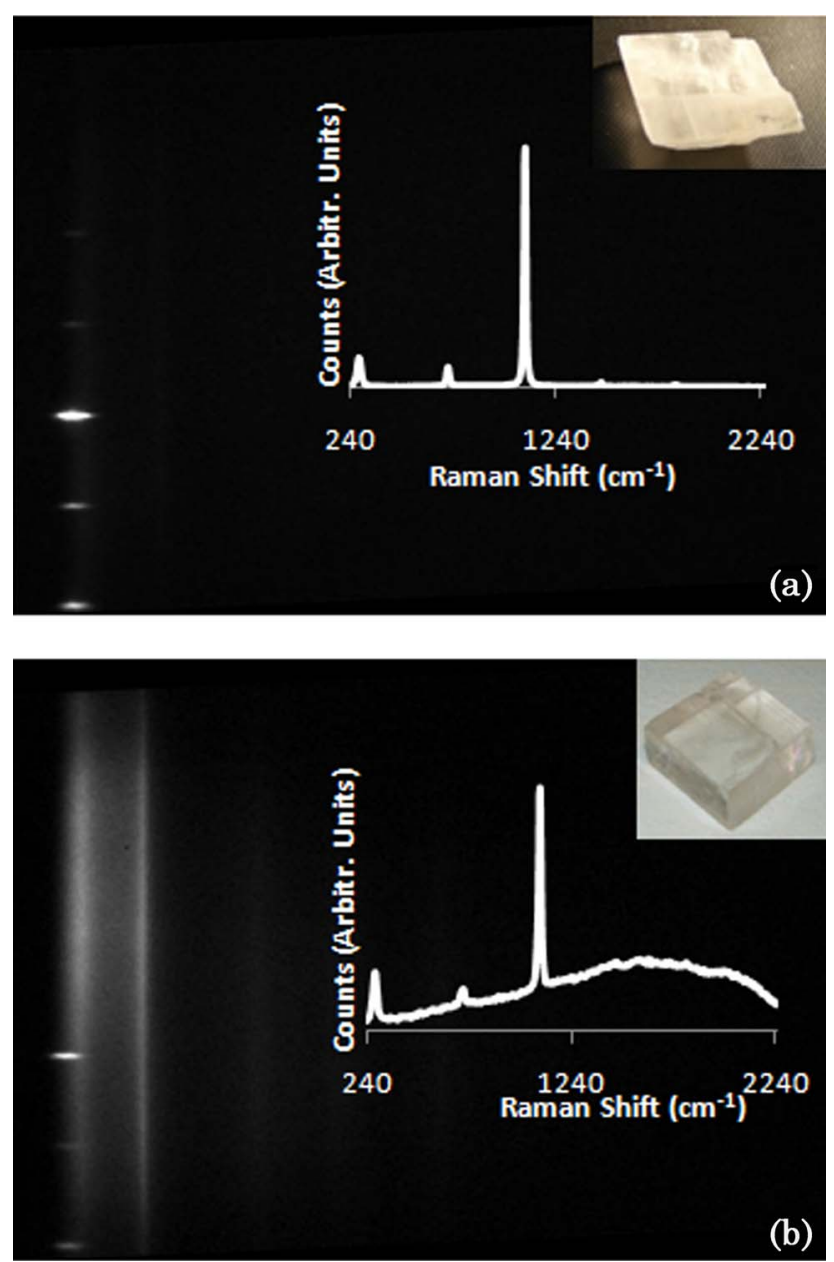

Fig. 2. (Color online) Streak camera images of calcite a) showing strong Raman return and b) showing Raman return and plasma effects. The horizontal scale covers $4 \mathrm{~ns}$, and the vertical scale covers $235-2600 \mathrm{~cm}^{-1}$. Insets show spectra averaged over time for the length of the laser pulse. Photos of each calcite sample show differences in opacity of the samples. Note that the oscillating nature of the plasma peak is an artifact of the streak camera sweep circuitry, causing small variations in sweep speed with time.

streaks in time. This spectrum was collected in synchroscan mode with a total integration time of $30 \mathrm{~s}$. In Fig. 2(b), a broadband peak is present after the laser pulse. This peak is likely emission from a plasma created near the sample surface. Figs. 2(a) and 2(b) show two different calcite samples, with one showing more plasma effects. One noticeable difference is that this calcite sample was flatter, smoother, and more transparent. The plasma effects are particularly noticeable in an opaque, smooth sample, such as silicon, where the entire laser pulse is absorbed in a very small sample volume, leading to high energy density.

Two-dimensional plots of intensity versus Raman shift shown as insets of Fig. 2 are created from the streak images by summing the counts over the time scale of the laser pulse width. This selects the Raman return and rejects the fluorescence. In cases in which the plasma emission is significant during the laser pulse, a background is still observed, although it is not fluorescence related. This is illustrated in the inset of Fig. 2(b), where the background is higher. It should be noted that without time resolution, this background would be indistinguishable from fluorescence, and important information relating to potential sample damage or alteration would be lost.

\section{A. Fluorescence Rejection}

We demonstrate fluorescence rejection using a variety of fluorescent minerals, some of which are highly relevant to the Martian environment. Montmorillonite, brushite, and magnesium sulfate are altered minerals that have been inferred on Mars, and all have exhibited strong fluorescence, leading to the inability to measure Raman spectra using a CW Raman system. These samples are representative of the most challenging fluorescence conditions that we can expect to find on Mars. In order to demonstrate the fluorescence rejection capabilities of our time-resolved Raman system, we have also performed measurements on the same samples using a Renishaw M1000 Micro Raman Spectrometer System with an Ar ion laser, $514.5 \mathrm{~nm}$ through an optical microscope. Both measurements were compared to spectra in the RRUFF database, as well as reports in the literature. The RRUFF samples are typically chosen with minimal fluorescence centers in order to generate the best Raman spectra for the database, and are not necessarily indicative of the expected fluorescence from a given mineral. Alternatively, many RRUFF spectra are taken using infrared excitation to avoid fluorescence in highly fluorescent samples.

\section{B. Willemite}

Willemite is a trigonal zinc silicate mineral $\left(\mathrm{Zn}_{2} \mathrm{SiO}_{4}\right)$ and a minor ore of zinc. Willemite is known for its strong green fluorescence peaked at $535 \mathrm{~nm}$ owing to the presence of $\mathrm{Mn}^{2+}$ [28]. The sample CIT1487 originated in Franklin, New Jersey. This mineral is highly fluorescent and provides a useful demonstration of the power of pulsed Raman under the most extreme fluorescence conditions. Figure 3 shows the time-resolved spectrum of willemite. As shown, the Raman spectrum from this sample was not measurable in the CW Raman system, even with only $1 \%$ of the laser power, due to saturation of the detector by fluorescence at all wavelengths. The time-resolved spectrum matches well with the RRUFF spectrum taken on an unoriented sample with a $785 \mathrm{~nm}$ excitation wavelength.

\section{Spodumene}

Spodumene is a monoclinic pyroxene mineral consisting of $\mathrm{LiAl}\left(\mathrm{SiO}_{3}\right)_{2}$. The origin of the sample 2919 is San Pedro Mine, Pala, California. The pink color of this sample is connected with $\mathrm{Mn}$ impurities responsible for strong fluorescence in the region of interest, saturating the detector at $1080 \mathrm{~cm}^{-1}$. The time-resolved spectrum in Fig. $\underline{4}$ matches well with 


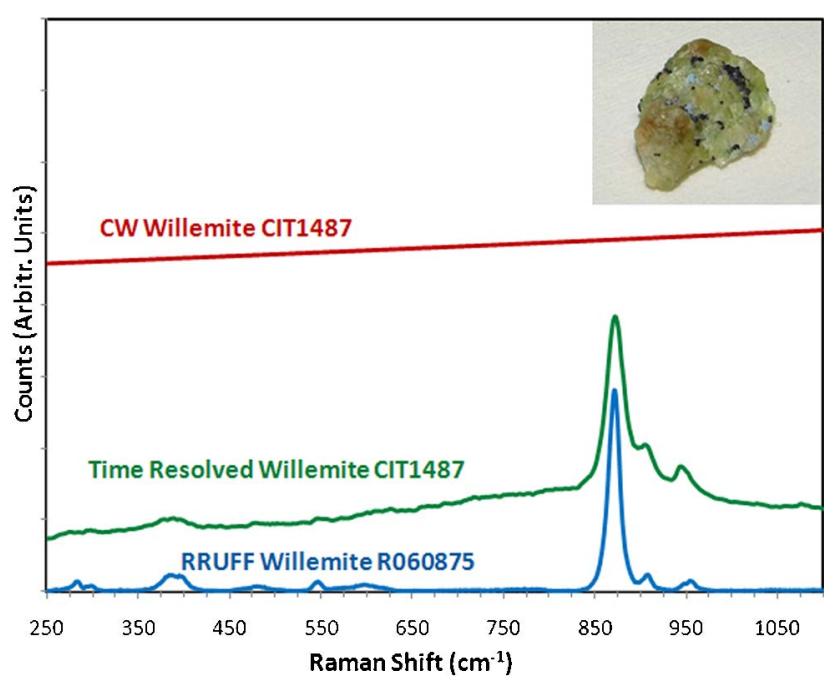

Fig. 3. (Color online) Raman spectra of willemite (pictured in the inset) obtained using pulsed and CW Raman. Under CW illumination, the fluorescence saturates the CCD detector. The RRUFF spectrum acquired using a $785 \mathrm{~nm}$ source is shown for reference.

the RRUFF spectrum taken on an unoriented sample with a $785 \mathrm{~nm}$ excitation wavelength.

\section{Montmorillonite}

Montmorillonite is a clay mineral of the smectite family and is a common alteration product of many types of rock. Spectroscopic data (e.g., MER APXS) supports the presence of clay minerals on Mars. Definitive data on these minerals would provide a model for the alteration of the martian surface supporting neutral- or slightly alkaline $\mathrm{pH}$. The Independence Class rocks of the Columbia Hills have undergone substantial alteration. The evidence suggests that they are dominated by montmorillonite or

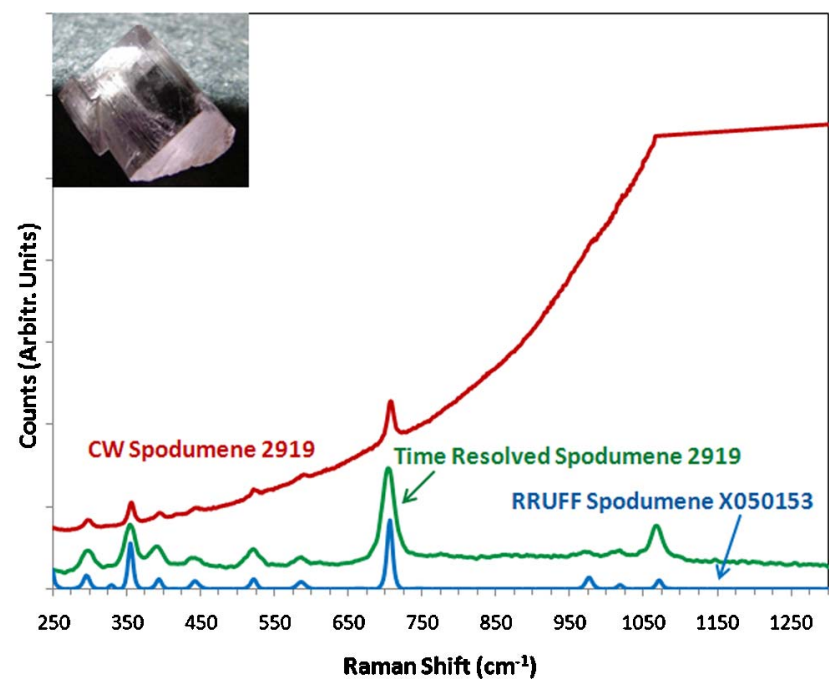

Fig. 4. (Color online) Raman spectra of spodumene (pictured in the inset) obtained using pulsed and CW Raman. The RRUFF spectrum acquired using a $785 \mathrm{~nm}$ source is shown for reference.

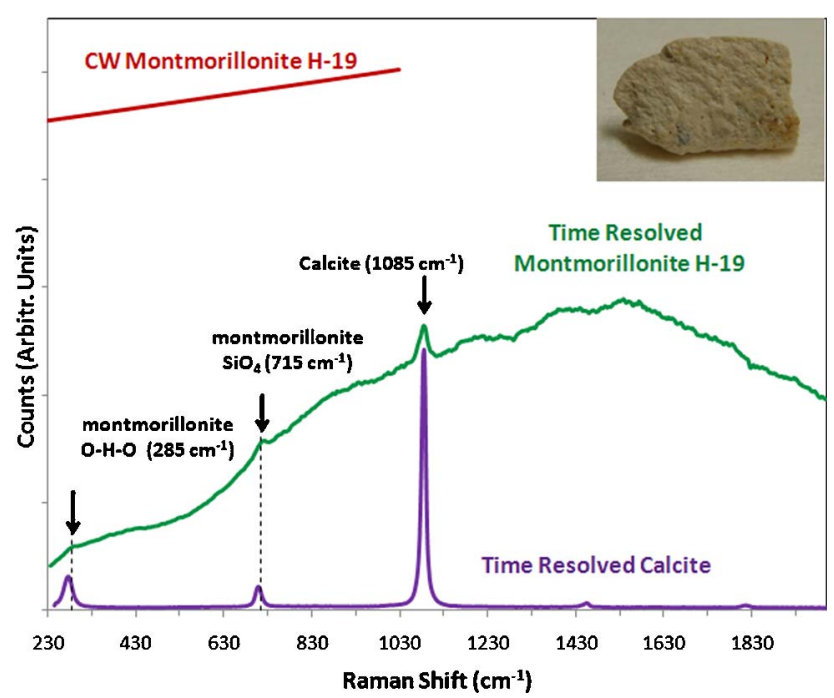

Fig. 5. (Color online) Raman spectra of montmorillonite (pictured in the inset) obtained using pulsed and CW Raman. Under CW illumination, the fluorescence saturates the CCD detector. A pulsed Raman spectrum of calcite is shown, as well, revealing that a minor phase of calcite is likely present.

its compositional equivalent [34]. Obtaining clean Raman spectra from clay minerals is highly challenging. They tend to be fine grained and powdery, with weak Raman bands owing to the small polarizability of Si-O bonds. They tend to be highly fluorescent, in part due to the presence of iron(III) hydroxide and organic matter [35]. We obtained Raman spectra from the Clay Mineral Standard H-19. The overwhelming fluorescence under $\mathrm{CW}$ excitation saturated the detector over the entire wavelength range. The time-resolved spectrum exhibited weak Raman peaks attributed to montmorillonite and a minor phase of calcite, as shown in Fig. 5. The most extensive Raman study of montmorillonite was performed using FT Raman with a $1064 \mathrm{~nm}$ laser to minimize fluorescence [36]. The strongest bands were reported in the $27 \overline{8-} 285 \mathrm{~cm}^{-1}$ range and the $708-725 \mathrm{~cm}^{-1}$ range. Variations are due to compositional differences between montmorillonite samples.

\section{E. Brushite}

Brushite is a phosphate mineral with a chemical formula $\mathrm{CaHPO}_{4} \cdot 2 \mathrm{H}_{2} \mathrm{O}$ that can be formed at low $\mathrm{pH}$ by reaction of phosphate-rich solutions with calcite and clay. It has been inferred on Mars in the Gusev Crater outcrops, rocks, and soils using MER APXS data [2]. We have observed strong fluorescence under CW excitation, saturating the CCD at $760 \mathrm{~cm}^{-1}$. The fluorescence background from the natural sample from Moorba Cave in Western Australia was too high to distinguish Raman peaks over the entire wavelength range. Time-resolved Raman revealed a Raman signature representative of brushite, as shown in Fig. $\underline{6}$. In addition to brushite, the presence of the anhydrous phase $\mathrm{CaHPO}_{4}$ is also hypothesized, based on the two strong peaks associated with the P-O stretching 


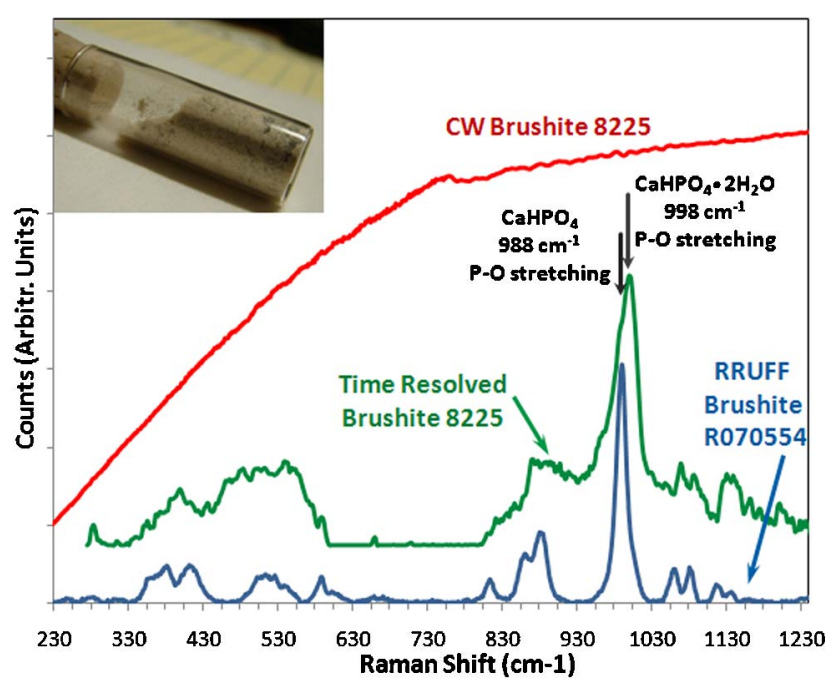

Fig. 6. (Color online) Raman spectrum of Brushite (loose powder pictured in the inset) obtained using pulsed and CW Raman. The time-resolved spectrum has been background subtracted. The RRUFF spectrum is also shown for reference. The peak locations of the P-O stretching modes for the anhydrous and hydrated phases from [37] are noted and reveal the presence of both phases.

modes at 988 and $998 \mathrm{~cm}^{-1}$ for the anhydrous and hydrated forms, respectively [37].

\section{F. Magnesium Sulfate}

Magnesium sulfate $\left(\mathrm{MgSO}_{4}\right)$ is hygroscopic and can exist in a range of hydration states, depending on its environmental history. Mg-sulfates are inferred on Mars, for example, in the Columbia Hills and Meridiani Planum outcrops [34]. Raman spectroscopy can provide a means to determine the degree of hydration, where Raman peaks shift to a higher wavenum-

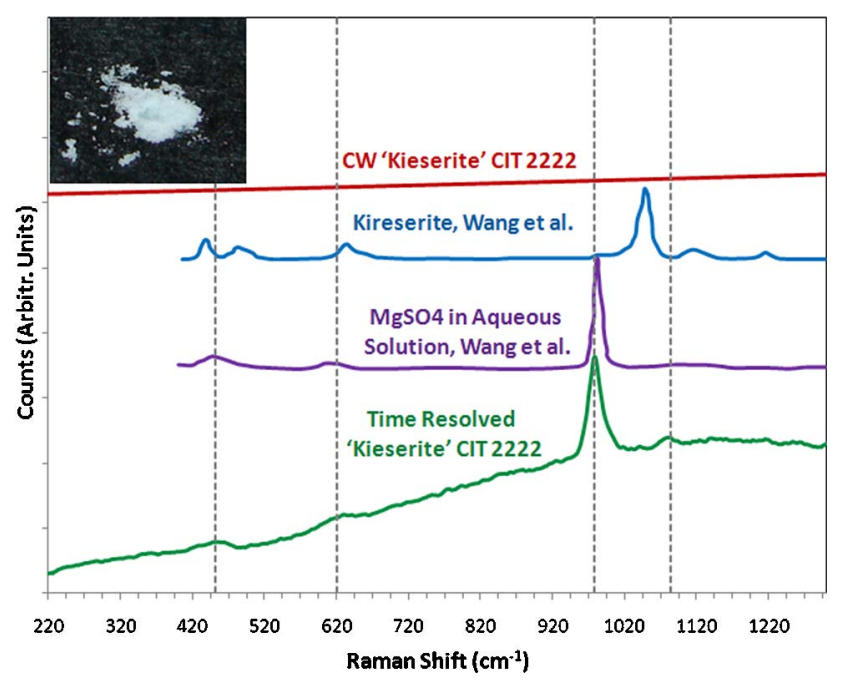

Fig. 7. (Color online) Raman spectrum of kieserite (loose white powder pictured in the inset) obtained using pulsed Raman. Under CW illumination, the fluorescence saturates the CCD detector. Data from [38] are plotted, as well, and show that the peaks shift to a higher wavenumber with decreasing hydration state. Our sample spectrum matches well with the completely hydrated sample. ber with decreasing hydration [38]. The origin of the sample 2222 is Stassfurt, Germany. CW Raman measurements of this kieserite sample revealed overwhelming fluorescence with no ability to detect Raman. Pulsed Raman revealed a fully hydrated sample, with peak positions coincident with those of $\mathrm{MgSO}_{4}$ in aqueous solution, as shown in Fig. 7 .

\section{Conclusions}

We have reviewed techniques for in situ mineralogy on planetary surfaces and presented time-resolved laser Raman spectroscopy as the next generation in situ instrument for definitive mineralogy of multiphase rock and soil samples preserving geological context. We have shown that time-resolved Raman spectroscopy is an excellent approach to identifying minerals when a high fluorescence background is present. This technique would have broad application to planetary mineralogy-for example, on Mars and its moons, Phobos and Deimos; Venus; Earth's moon; and asteroids. In particular, it is recommended for an in situ instrument on Mars, where fluorescence is likely to be of concern. For Mars, the objectives for a proposed 2018 mission point to a potential landing site with access to outcrops of diverse mineralogy where ancient habitability is hypothesized, and a possibility exists for detecting organics and biosignatures $[39,40]$. This suggests a heavily altered region with secondary minerals, many of which are similar to those chosen for this work (e.g., clays, sulfates, and phosphates). Our demonstration of strong fluorescence interference in these samples is motivation for selecting a time-resolved Raman instrument that operates regardless of fluorescence background. The comprehensive data required for potential sample return preselection would be acquired by mapping the mineralogy of a sample using timeresolved Raman spectroscopy. Mapping of the mineralogy is crucial if we can hope to make sense of complementary data on trace ions and organics within the context of the host rock. Such data would be obtained by other instruments on the same payload, such as time-resolved fluorescence spectroscopy for identifying trace ions, and ultraviolet fluorescence spectroscopy for mapping of organics. With the emphasis in planetary exploration shifting toward possible sample return from Mars, the Moon, Venus, and asteroids, Raman spectroscopy is expected to become an essential tool for in situ mineralogical analysis. It would be important in preselection for sample caching and potential return to Earth, as well as nondestructive high resolution measurement on samples after return to Earth.

We acknowledge invaluable discussions on Mars 2018 with Sabrina Feldman at the Jet Propulsion Laboratory (JPL). The research described in this publication was carried out at the Jet Propulsion Laboratory, California Institute of Technology, under a contract with the National Aeronautics and Space Administration (NASA). Continuous-wave Raman measurements were performed at the Mineral 
Spectroscopy Laboratory in the Department of Geological and Planetary Sciences at the California Institute of Technology, and time-resolved experiments at the JPL.

\section{References}

1. R. L. McCreery, Raman Spectroscopy for Chemical Analysis (Wiley, 2000).

2. D. W. Ming, R. V. Morris, and B. C. Clark, "Aqueous alteration on Mars," in The Martian Surface: Composition, Mineralogy and Physical Properties, J. F. Bell III, ed. (Cambridge University Press, 2008).

3. L. A. Soderblom and J. F. Bell III, "Exploration of the Martian surface: 1992-2007," in The Martian Surface: Composition, Mineralogy and Physical Properties, J. F. Bell III, ed. (Cambridge University Press, 2008).

4. S. Murchie, R. Arvidson, P. Bedini, K. Beisser, J.-P. Bibring, J. Bishop, J. Boldt, P. Cavender, T. Choo, R. T. Clancy, E. H. Darlington, D. Des Marais, R. Espiritu, D. Fort, R. Green, E. Guinness, J. Hayes, C. Hash, K. Heffernan, J. Hemmler, G. Heyler, D. Humm, J. Hutcheson, N. Izenberg, R. Lee, J. Lees, D. Lohr, E. Malaret, T. Martin, J. A. McGovern, P. McGuire, R. Morris, J. Mustard, S. Pelkey, E. Rhodes, M. Robinson, T. Roush, E. Schaefer, G. Seagrave, F. Seelos, P. Silverglate, S. Slavney, M. Smith, W.-J. Shyong, K. Strohbehn, H. Taylor, P. Thompson, B. Tossman, M. Wirzburger, and M. Wolff, "Compact reconnaissance imaging spectrometer for Mars (CRISM) on Mars reconnaissance orbiter (MRO)," J. Geophys. Res. [Planets] 112(E5), E05S03 (2007).

5. A. S. McEwen, E. M. Eliason, J. W. Bergstrom, N. T. Bridges, C. J. Hansen, W. A. Delamere, J. A. Grant, V. C. Gulick, K. E. Herkenhoff, L. Keszthelyi, R. L. Kirk, M. T. Mellon, S. W. Squyres, N. Thomas, and C. M. Weitz, "Mars reconnaissance orbiter's high resolution imaging science experiment (HiRISE)," J. Geophys. Res. [Planets] 112(E5), E05S02 (2007).

6. P. R. Christensen, M. B. Wyatt, T. D. Glotch, A. D. Rogers, S. Anwar, R. E. Arvidson, J. L. Bandfield, D. L. Blaney, C. Budney, W. M. Calvin, A. Fallacaro, R. L. Fergason, N. Gorelick, T. G. Graff, V. E. Hamilton, A. G. Hayes, J. R. Johnson, A. T. Knudson, H. Y. McSween, G. L. Mehall, L. K. Mehall, J. E. Moersch, R. V. Morris, M. D. Smith, S. W. Squyres, S. W. Ruff, and M. J. Wolff, "Mineralogy at Meridiani Planum from the Mini-TES experiment on the Opportunity Rover," Science 306, 1733-1739 (2004).

7. P. R. Christensen, J. L. Bandfield, R. N. Clark, K. S. Edgett, V. E. Hamilton, T. Hoefen, H. H. Kieffer, R. O. Kuzmin, M. D. Lane, M. C. Malin, R. V. Morris, J. C. Pearl, R. Pearson, T. L. Roush, S. W. Ruff, and M. D. Smith, "Detection of crystalline hematite mineralization on Mars by the Thermal Emission Spectrometer: evidence for near-surface water," J. Geophys. Res. [Planets] 105, 9623-9642 (2000).

8. J. P. Grotzinger, R. E. Arvidson, J. F. Bell, W. Calvin, B. C. Clark, D. A. Fike, M. Golombek, R. Greeley, A. Haldemann, K. E. Herkenhoff, B. L. Jolliff, A. H. Knoll, M. Malin, S. M. McLennan, T. Parker, L. Soderblom, J. N. Sohl-Dickstein, S. W. Squyres, N. J. Tosca, and W. A. Watters, "Stratigraphy and sedimentology of a dry to wet eolian depositional system, Burns formation, Meridiani Planum, Mars," Earth Planet. Sci. Lett. 240, 11-72 (2005).

9. P. Mahaffy, "Sample analysis at Mars: developing analytical tools to search for a habitable environment on the red planet," Geochem. News 141 (2009).

10. A. Wang, B. L. Jolliff, and L. A. Haskin, "Investigating surface mineralogy, alteration processes, and biomarkers on Mars using laser Raman spectroscopy," in Sixth International Conference on Mars, abstract no. 3270 (2003).

11. M. C. Storrie-Lombardi, J-P. Muller, M. R. Fisk, C. Cousins, B. Sattler, A. D. Griffiths, and A. J. Coates, "Laser-induced fluorescence emission (L.I.F.E.): searching for Mars organics with a UV-enhanced PanCam," Astrobiology 9(10), 953-964 (2009).

12. A. T. Basilevsky, M. A. Ivanov, J. W. Head, M. Aittola, and J. Raitala, "Landing on Venus: past and present," Planet. Space Sci. 55, 2097-2112 (2007).

13. M. Y. Zolotov, B. Fegley, Jr., and K. Lodders, "Hydrous silicates and water on Venus," Icarus 130, 475-494 (1997).

14. A. J. Ball, M. E. Price, R. J. Walker, G. C. Dando, N. S. Wells, and J. C. Zarnecki, "Mars Phobos and Deimos survey (M-PADS)-A Martian Moons orbiter and Phobos lander," Adv. Space Res. 43, 120-127 (2009).

15. E. M. Galimov, "Phobos sample return mission: scientific substantiation," Sol. Syst. Res. 44(1), 5-14 (2010).

16. A. Wang, B. L. Jolliff, and L. A. Haskin, "Raman spectroscopy as a method for mineral identification on lunar robotic exploration missions," J. Geophys. Res. 100(E10), 21189-21199 (1995).

17. RRUFF Project: http://rruff.info/.

18. T. Hirschfeld and B. Chase, "FT-Raman spectroscopy: development and justification," Appl. Spectrosc. 40 (2), 133-137 (1986).

19. B. Chase, "Fourier transform Raman spectroscopy," J. Am. Chem. Soc. 108, 7485-7488 (1986).

20. L. Burgio and R. J. H. Clark, "Library of FT-Raman spectra of pigments, minerals, pigment media and varnishes, and supplement to existing library of Raman spectra of pigments with visible excitation," Spectrochim. Acta Part A 57, 1491-1521 (2001).

21. E. B. Hanlon, R. Manoharan, T-W. Koo, K. E. Shafer, J. T. Motz, M. Fitzmaurice, J. R. Kramer, I. Itzkan, R. R. Dasari, and M. S. Feld, "Prospects for in vivo Raman spectroscopy," Phys. Med. Biol. 45, R1-R59 (2000).

22. Y. Wang and R. L. McCreery, "Evaluation of a diode laser/ charge coupled device spectrometer for near-infrared Raman spectroscopy," Anal. Chem. 61, 2647-265 (1989).

23. H. G. M. Edwards, S. E. Jorge Villar, J. Jehlicka, and T. Munshi, "FT-Raman spectroscopic study of calcium-rich and magnesium-rich carbonate minerals," Spectrochim. Acta Part A 61, 2273-2280 (2005).

24. P. Makreski and G. Jovanovski, "Minerals from Macedonia. XXII. Laser-induced fluorescence bands in the FT-Raman spectrum of almandine mineral," J. Raman Spectrosc. 39, 1210-1213 (2008).

25. A. Aminzadeh, "Fluorescence bands in the FT-Raman spectra of some calcium minerals," Spectrochim. Acta Part A 53 693-697 (1997)

26. T. Frosch, N. Tarcea, M. Schmitt, H. Thiele, F. Langenhorst, and J. Poppet, "UV Raman imaging: a promising tool for astrobiology: comparative Raman studies with different excitation wavelengths on SNC Martian meteorites," Anal. Chem. 79, 1101-1108 (2007).

27. N. M. Johnson and B. Fegley, "Longevity of fluorine-bearing tremolite on Venus," Icarus 165(2), 340-348 (2003).

28. M. Gaft, R. Reisfeld, and G. Panczer, Modern Luminescence Spectroscopy of Minerals and Materials (SpringerVerlag, 2005).

29. M. Gaft and L. Nagli, "Gated Raman spectroscopy: potential for fundamental and applied mineralogy," Eur. J. Mineral. 21, 33-42 (2009).

30. T. Tahara and H. Hamaguchi, "Picosecond Raman spectroscopy using a streak camera," Appl. Spectrosc. 47(4), 391-398 (1993). 
31. N. Everall, T. Hahn, P. Matousek, A. W. Parker, and M. Towrie, "Picosecond time-resolved Raman spectroscopy of solids: capabilities and limitations for fluorescence rejection and the influence of diffuse reflectance," Appl. Spectrosc. 55, 1701-1708 (2001).

32. H. Hamaguchi and T. L. Gustafson, "Ultrafast time-resolved spontaneous and coherent Raman spectroscopy: the structure and dynamics of photogenerated transient species," Annu. Rev. Phys. Chem. 45, 593-622 (1994).

33. R. L. McCreery, "Photometric standards for Raman spectroscopy," in Handbook of Vibrational Spectroscopy, J. M. Chalmers and P. R. Griffiths, eds. (Wiley, 2002).

34. S. W. Ruff, P. R. Christensen, T. D. Glotch, D. L. Blaney, J. E. Moerch, and M. B. Wyatt, "The mineralogy of Gusev crater and Meridiani Planum derived from the Miniature Thermal Emission Spectrometers on the Spirit and Opportunity rovers," in The Martian Surface: Composition, Mineralogy and Physical Properties, J. F. Bell III, ed. (Cambridge University Press, 2008)

35. J. M. Alia, H. G. M. Edwards, F. J. Garcia-Navarro, J. Parras-Armenteros, and C. J. Sanchez-Jimenez, "Applica- tion of FT-Raman spectroscopy to quality control in brick clays firing process," Talanta 50, 291-298 (1999).

36. R. L. Frost and L. Rintoul, "Lattice vibrations of montmorillonite: an FT Raman and x-ray diffraction study," Appl. Clay Sci. 11, 171-183 (1996).

37. J. Xu, I. S. Butler, and D. F. R. Gilson, "FT-Raman and highpressure infrared spectroscopic studies of dicalcium phosphate dihydrate $\left(\mathrm{CaHPO}_{4} \cdot 2 \mathrm{H}_{2} \mathrm{O}\right)$ and anhydrous dicalcium phosphate $\left(\mathrm{CaHPO}_{4}\right)$," Spectrochim. Acta Part A 55, 2801-2809 (1999).

38. A. Wang, J. F. Freeman, B. L. Jolliff, and I-M Chou, "Sulfates on mars, a systematic Raman spectroscopic study of hydration states of magnesium sulfates," Geochim. Cosmochim. Acta 7024, 6118-6135 (2006).

39. P. Christensen, "Scientific overview of Mars sample return," presented at the 22nd MEPAG Meeting, Monrovia, California, 17-18 March 2010.

40. C. G. Salvo and A. Elfving, "Proposed Mars astrobiology explorer-Cacher (MAX-C) \& ExoMars 2018 (MXM-2018) Mission Formulation Status," presented at the 22nd MEPAG Meeting, Monrovia, California, 17-18 March 2010. 